AperTO - Archivio Istituzionale Open Access dell'Università di Torino

\title{
Gradus realitatis - die intensive Größe bei Kant und im Neukantianismus
}

\section{This is the author's manuscript}

Original Citation:

Availability:

This version is available http://hdl.handle.net/2318/1737660

since 2020-04-29T10:34:45Z

Publisher:

De Gruyter

Published version:

DOI:10.1515/9783110345148-010

Terms of use:

Open Access

Anyone can freely access the full text of works made available as "Open Access". Works made available under a Creative Commons license can be used according to the terms and conditions of said license. Use of all other works requires consent of the right holder (author or publisher) if not exempted from copyright protection by the applicable law. 


\section{Gradus realitatis - die intensive Größe bei Kant und im Neukantianismus}

\section{Kants Grundsatz der Antizipationen der Wahrnehmung}

Die Beschäftigung mit dem Thema „intensive Größe“ bei Kant und im Neukantianismus bedeutet vor allem, über Kants Grundsatz der „Anticipationen der Wahrnehmung“ (vgl. B207-218) und seine Rezeption zu reden. Dieser Grundsatz ist der zweite der in der Kritik der reinen Vernunft aufgelisteten Grundsätze des reinen Verstandes. „In allen Erscheinungen“, so formuliert ihn Kant, „hat das Reale, was ein Gegenstand der Empfindung ist, intensive Größe, d. i. einen Grad“ (B207). Auf den ersten Blick erscheint dies als eine ziemlich rätselhafte Aussage. Was heißt „Antizipation“? Was ist unter dem Ausdruck „das Reale“ zu verstehen? Wie soll der Bezug auf die Empfindung interpretiert werden? Wie kann man behaupten, dass ihm ein Grad oder eine intensive Größe zugeschrieben werden muss? Der erste Teil der vorliegenden Arbeit versucht auf diese Fragen so weit wie möglich eine Antwort zu finden, ohne damit den Anspruch zu erheben, alle Interpretationsprobleme lösen zu können. Die Rezeption von Kants Grundsatz in der nachkantischen Philosophie und insbesondere im Neukantianismus, die im zweiten Teil dargestellt wird, soll dazu beitragen, außer der rein exegetischen Frage die theoretische Tragweite dessen zu verstehen, was man mit Recht „den vergessenen Grundsatz"1 genannt hat.

\subsection{Antizipation, Empfindung, Wahrnehmung und Realität}

Kant definiert die Antizipation als jene „Erkenntniß, wodurch ich dasjenige, was zur empirischen Erkenntniß gehört, a priori erkennen und bestimmen kann“ (B208). Die Antizipation ist also zunächst eine Erkenntnis a priori, d.h. völlig unabhängig von jeder möglichen Erfahrung. Eine solche Erkenntnis a priori betrifft - gemäß den Prinzipien der transzendentalen Philosophie - selbstver-

1 Vgl. Theodore E. Uehling, „The Forgotten Principle: Kant's Anticipations of Perception,“ in Akten des 5. Internationalen Kant-Kongresses Mainz. April 4-8, 1981, hrsg. von Gerhard Funke (Bonn: Bouvier, 1981). 
ständlich nicht die „Gegenstände[...]“, sondern „unsere[...] Erkenntnißart von Gegenständen“ (B25). Was aber die Antizipationen gegenüber anderen Erkenntnissen a priori charakterisiert, ist, dass sie sogar den Anspruch erheben, etwas über unsere „empirische Erkenntnis“ (B208) a priori festzustellen. Die Antizipationen versuchen nämlich, „dasjenige a priori vor[zu]stellen, was immer a posteriori in der Erfahrung gegeben werden mag“ (B209). Das bedeutet für Kant in erster Linie etwas a priori über „die Empfindung (als Materie der Wahrnehmung)“ (B209) zu behaupten. Wenn man nämlich etwas finden würde, das „sich an jeder Empfindung als Empfindung überhaupt (ohne daß eine besondere gegeben sein mag) a priori erkennen läßt: so würde dieses im ausnehmenden Verstande Anticipation genannt zu werden verdienen“ (B209).

Kant selbst ist sich bewusst, dass der Anspruch, etwas a priori über die Empfindung zu behaupten, „befremdlich scheint“ (B209). Die Empfindung ist doch par excellence das, das ,an den Erscheinungen [...] niemals a priori erkannt wird“ (B208). Die Empfindung stellt sogar „den eigentlichen Unterschied des Empirischen von dem Erkenntniß a priori“ dar (B209): „so folgt, daß diese es eigentlich sei, was gar nicht anticipirt werden kann“ (B209). Damit, so weiter Kant, ist es „etwas Auffallendes an sich und erregt darüber einiges Bedenken, daß der Verstand“ etwas von der Empfindung „,anticipiren könne“ (B217). Nichtsdestotrotz behauptet Kant, „,so verhält es sich hier wirklich“ (B209). Etwa zehn Jahre später (April 1800) bestätigt Kant seine Überzeugung in ganz ähnlichen Worten:

Es ist befremdlich; es scheint gar unmöglich zu seyn das was auf Wahrnehmungen (empirischen Vorstellungen mit Bewustseyn derselben) beruht a priori darstellen zu wollen z. B. den Schall, das Licht, die Wärme etc welche ins gesammt das Subjective [...] der Warnehmung d.i. der empirischen Vorstellung mit Bewustseyn ist [,] mithin kein Erkentnis eines Objects bey sich führt; und doch ist dieser Act des Vorstellungsvermögens nothwendig. (AA 22:493)

Um die Natur dieses paradoxen Anspruchs zu erklären, etwas a priori über das zu behaupten, was a posteriori par excellence ist, nämlich die Empfindung, muss man zunächst festhalten, dass Kant eigentlich von „Antizipationen der Wahrnehmung“ und nicht direkt von „Antizipationen der Empfindung“ spricht. Wahrnehmung und Empfindung, wenn auch eng miteinander verbunden, scheinen nicht identisch zu sein. Kant definiert üblicherweise die „Wahrnehmung“ als die „Empfindung, deren man sich bewußt ist“ (B272). ${ }^{2}$ Die Wahrnehmung ist nämlich das

2 Vgl. auch diese Stellen: „Wahrnehmung ist das empirische Bewußtsein, d.i. ein solches, in welchem zugleich Empfindung ist“ (Kant, Kritik der reinen Vernunft [KrV, im Folgenden mit A oder B ohne „KRV“ zitiert], B207). „[D]ie Anschauung, deren ich mir bewußt bin, d.i. Wahrnehmung (perceptio)“ (Immanuel Kant: Gesammelte Schriften, herausgegeben von der Preussischen Aka- 
Bewusstsein von jener Wirkung eines Gegenstandes auf die Sinne, die man Empfindung nennt (vgl. B34). Die Empfindung ist also bloß die „Materie der Wahrnehmung“ (B209), die mit dieser nicht völlig identisch ist. Sind in der Empfindung der Zustand des Empfindenden und das Empfundene sozusagen eins, so scheint dagegen die Wahrnehmung auf etwas $\mathrm{zu}$ verweisen, das die Empfindung verursacht, aber von dieser verschieden ist. Eine relativ späte Reflexion (1790 - 1804) erklärt Kants Standpunkt am besten:

Das Bewustseyn von der Gegenwart eines Gegenstandes ist Warnehmung. - Das Subjective der Warnehmung ist Empfindung, das Objective, d.i. der Begrif des Empfundenen, ist Realitaet. (AA 28:654, Refl.)

Die Wahrnehmung besitzt also einen subjektiven Aspekt, nämlich die Empfindung, und einen objektiven, d. h. das, was ,mich afficirt, und diese Empfindung in mir hervorbringt“ (AA 12:174; vgl. auch B207). Dies bezeichnet Kant mit dem Wort „Realität“. Was antizipiert werden kann ist also nicht bloß Empfindung, sondern etwas komplexeres: „Wahrnehmung (Empfindung und mithin Realität)“ (B212).

In der zweiten Auflage der Kritik der reinen Vernunft wird der Gegenstand der Antizipationen also als „das Reale, was ein Gegenstand der Empfindung ist“ (B207) bezeichnet; Kant formulierte dies in der ersten Auflage noch etwas anders: „die Empfindung und das Reale, welches ihr an dem Gegenstande entspricht, (realitas phaenomenon)“(A166). Es ist plausibel, dass der Grund dieser Änderung darin besteht, dass Kant eine psychologische Interpretation des Grundsatzes vermeiden wollte, als ob die bloße Empfindung als psychophysiologische Tatsache der Gegenstand der Antizipationen wäre. Das Objekt der Antizipationen scheint vielmehr in der Verbindung zwischen Empfindung und Realität zu bestehen, obwohl Kant in seinem Sprachgebrauch nicht immer konsequent ist. ${ }^{3}$

Wenn man Kant nicht eine Form von Psychologismus vorwerfen möchte, kann man dem Bezug auf die Empfindung eine restriktive Bedeutung zuschreiben: Das, was antizipiert werden kann, ist nicht die „Realität“ als solche, sondern die Realität als das, „was einer Empfindung überhaupt correspondirt“, d.h. die „realitas phaenomenon“ (B182). Das bedeutet implizit jedoch, dass über die „Realität[, die] nur durch den reinen Verstand vorgestellt wird (realitas noumenon)“ (B329), nichts a priori gesagt werden darf.

demie der Wissenschaften, Berlin $1900 \mathrm{ff} .(=\mathrm{AA}$; AA 4:300). „Das Bewußtsein einer empirischen Anschauung heißt Wahrnehmung“ (AA 8:217).

3 Vgl. dazu Luigi Scaravelli, Saggio sulla categoria della realtà (Florenz: Le Monnier, 1947), 170. 


\subsection{Realitas phaenomen und realitas noumenon}

Was heißt aber für Kant „Realität“? Kant versteht unter „Realität“ nicht die Existenz oder Wirklichkeit, sondern die qualitative Bestimmung, die den Gegenstand qua Etwas, Sache oder Ding qualifiziert. Das lateinische Wort realitas stammt von res (Ding, Sache) ab und Kant umschreibt „Realität“ gelegentlich mit „Sachheit“ oder „Dingheit“, die als buchstäbliche Übersetzungen von realitas betrachtet werden können: „Realitas kann eigentlich nicht auf Deutsch gegeben werden. Eigentlich bedeutet es Dingheit, Sachheit“ (AA 28:1146). Real zu sein ist also gleichbedeutend mit Etwas-sein, im Gegensatz zum Nichts-sein. Die Realität definiert ein Etwas als ein solches, indem es dieses von etwas anderem, das es nicht ist, abgrenzt. Das Gegenteil von „Realität“ ist daher nicht, wie im alltäglichen Sprachgebrauch, Schein oder Illusion, sondern „Negation“: „Der Unterschied unter der Realität und Negation ist: Realität ist, dessen Begriff ein Seyn in sich enthält; Negation, dessen Begriff ein Nichtseyn in sich enthält“ (AA 28:552). Realität und Negation sind also die allgemeinen positiven oder negativen Prädikate, die einem Ding zugeschrieben werden können, und sie haben deswegen eine Verbindung mit dem affirmativen und negativen Urteil. Kant überträgt also die in der Logik übliche Bezeichnung „Qualität“ für den affirmativen oder negativen Charakter des Urteils auf die Kategorien Realität und Negation, die als „Qualitätskategorien“ bezeichnet werden (vgl. B97f.; B106ff.).

Kant übernimmt diesen Sprachgebrauch aus den Lehrbüchern von Christian Wolff und Alexander Baumgarten ${ }^{4}$, ,worin die Überlieferung der mittelalterlichen und neuzeitlichen Metaphysik schulmäßig verarbeitet ist“. ${ }^{5}$ Kant also „hält zunächst die Schuldefinition von Realität und Negation fest; ${ }^{6}$ aber „,[w]ie allen aus der überlieferten Metaphysik aufgenommenen Grundbegriffen gibt Kant auch

\footnotetext{
4 In seiner Ontologia betrachtet Christian Wolff realitas als Synonym von quidditas: „quidquid est vel esse posse concipitur, dicitur res, quatenus est aliquid; ut adeo res definiri possit per id, quod est aliquid. Unde realitas et quidditas apud scholasticos synonyma sunt“ Christian Wolff, „Philosophia prima sive Ontologia (1730),“ in Ders., Gesammelte Werke, hrsg. von Jean Ecole (Hildesheim: Olms, 1962), Bd. II.3, §243. Baumgarten bezeichnet als Realitäten auch die positiven Prädikate, die einem Wesen zugeschrieben werden können: „quae determinando ponuntur in aliquo (notae et praedicata), sunt DETERMINATIONES, altera positiva, et affirmativa, quae si vere sit, est REALITAS, altera negativa, quae si vere sit, est NEGATIO“ Alexander Gottlieb Baumgarten, Metaphysica, 2 ed. (Halle: Hemmerde, 1743), §36.

5 Martin Heidegger, „Die Frage nach dem Ding. Zu Kants Lehre von den transzendentalen Grundsätzen,“ in Gesamtausgabe, hrsg. von Friedrich-Wilhelm von Herrmann (Klostermann, 1984: Franfurt am Main), Bd. 41, 216.
}

6 Anneliese Maier, Kants Qualitätskategorien (Berlin: Metzner, 1930), 29. 
diesem, der realitas, eine neue kritische Auslegung “. ${ }^{7}$ Mit anderem Worten: Kant dehnt „die Unterscheidung von phaenomenon und noumenon auch auf den Realitätsbegriff“ aus: ${ }^{8}$

Die realitas ist entweder phaenomenon, oder noumenon. Alles, was sich positiv unsern Sinnen darstellt, heißt: realitas phaenomenon; und alles, was sich positiv unserm reinen Verstande darstellt, ist realitas noumenon. Realitas phaenomenon oder Realität in der Erscheinung (oder Schein-Realität) ist die, die nur in unsern Sinnen liegt (AA 28:559f.).

In den Erscheinungen ist also „Realität [...] das Empfindbare“ (AA 29:715) (sensibile), in den Noumena ist sie das „Denkbare nach Begriffen“ (cogitabile). Die Antizipationen der Wahrnehmung haben den Anspruch, bloß über die realitas phaenomenon, die „Realität in der Erscheinung“, die Realität, die Objekt der Empfindung ist, etwas a priori zu behaupten; die realitas noumenon bezieht sich dagegen auf die Realität, sofern sie durch den bloßen Verstand gedacht wird, und sie findet bloß in der Transzendentalen Dialektik (B599ff.) in Bezug auf das ens realissimum Anwendung, worüber aber keine Aussage a priori gemacht werden darf.

Die Vermittlung und die Unterscheidung zwischen diesen zwei Bedeutungen der Realitätskategorie findet, wie für jede andere Kategorie, im „Schematismuskapitel“ statt. Realität und Negation werden hier auf das Schema „einer erfüllten oder leeren Zeit“ (B182) restringiert, das in concreto in der Erfahrung gegeben werden darf. ${ }^{9}$ Dagegen kann von Realität und Negation als reinen Kategorien „kein correspondirendes Schema der Sinnlichkeit gegeben werden“, so dass sie „keinen Gegenstand in concreto haben können“ (B692). Man kann also behaupten, dass die realitas phaenomenon bloß „ein Etwas bedeutet, das im Raume und der Zeit angetroffen wird“ (B329). ${ }^{10}$ Dagegen bezieht sich die realitas noumenon auf ein „Etwas überhaupt“, wie es nach bloßen Begriffe gedacht werden kann.

7 Heidegger, „Die Frage nach dem Ding. Zu Kants Lehre von den transzendentalen Grundsätzen,“ 216.

8 Maier, Kants Qualitätskategorien, 33.

9 Vgl. dazu Bruno Haas, „Kants Qualitätsschematismus, “ in Analysen - Probleme - Kritik 1, hrsg. von Hariolf Oberer (Würzburg, Germany: Königshausen und Neumann, 1988).

10 „Die qvalitaet eines Dinges [als Dinges], die es [von] als ein Etwas von der bloßen Form unterscheidet, ist Realität, und ihr correspondirt Empfindung“ (AA 18:663). 


\subsection{Gradus rationis und gradus perfectionis}

Was kann von der Realität, die der Empfindung korrespondiert, antizipiert, d.h. $a$ priori behauptet, werden? Kants Antwort ist eindeutig: „Die Qualität der Empfindung ist jederzeit bloß empirisch und kann a priori gar nicht vorgestellt werden (z.B. Farben, Geschmack etc.)“ (B217); die Natur der Sinnesempfindungen (das, was z.B. Wärme von Farben unterscheidet) hängt nämlich von der zufälligen biologischen Struktur des Menschen ab und kann nicht $a$ priori bestimmt werden (vgl. z. B. AA 5:224), „die Eigenschaft“ der Empfindungen dagegen, „,aß sie einen Grad haben, kann a priori erkannt werden“ (B218). Hierzu heißt es in den Prolegomena:

mithin, obzwar Empfindung als die Qualität der empirischen Anschauung in Ansehung dessen, worin sie sich specifisch von andern Empfindungen unterscheidet, niemals a priori erkannt werden kann, sie dennoch in einer möglichen Erfahrung und überhaupt als Größe der Wahrnehmung intensiv von jeder andern gleichartigen unterschieden werden könne (AA 4:309).

Die Axiome der Anschauung, die ersten der von Kant aufgelisteten Grundsätze, behaupten a priori, dass alle Erscheinungen, sofern sie Raum und Zeit einnehmen, extensive Größen sind; die „Anticipationen der Wahrnehmung“ behaupten gleichermaßen a priori, dass die Empfindungsqualitäten (Wärme, Dichte, Bewegung usw.), die Raum und Zeit mehr oder weniger erfüllen, einen Grad oder eine intensive Größe haben. Kant kann also ganz unabhängig von jeder Erfahrung behaupten: „Alles in der Erscheinung ist quantum, so wohl so fern es Zeit oder Raum enthält (extensive tale), oder sie erfüllt, d.i. in der Zeit oder dem Raum enthalten ist (intensive tale: realitaet in der Empfindung)“ (AA 18:409). ${ }^{11}$

Eine solche Aussage hat einerseits eine wissenschaftstheoretische Bedeutung: Sie ermöglicht es, „die Mathematik auf Erscheinungen anzuwenden“ (B221). Außer der „Mathematik der Ausdehnung“ (B204) (mathesis extensorum: Geometrie oder Phoronomie), die Raum und Zeit betrifft, gibt es nämlich auch eine „zweite Anwendung der Mathematik auf Naturwissenschaft“" (mathesis intensorum), die die Empfindungsqualitäten betrifft: „daher [können] sowohl bei der einen, als bei der andern die Zahlgrößen und mit ihnen die Bestimmung der Erscheinung als Größe gebraucht werden“ (B222).

11 „Alle Erscheinung hat als Anschauung ihre extensive Große und als Empfindung ihren Grad“ (AA 18: 41, Refl. 5585); „Alles, was im Raum und in der Zeit vorgestellt wird, hat extensive Größe. Alle Realität im Raume und in der Zeit hat einen Grad“ (AA 28:562). 
Indem aber Kant nicht einfach von Empfindungsqualitäten spricht, sondern den Begriff „Realität“ verwendet, verweist er gleichzeitig auf eine ontologische Tradition, in der der Begriff von „Grad“ und „Realität“ eine metaphysische Bedeutung annimmt, von der er Abstand nehmen will. Wenn man nämlich die realitas phaenomenon mit ihrer ontologischen Definition, die in dem Begriff realitas noumenon enthalten ist, vergleicht, sieht man, dass die kritische Wende darin besteht, „dass der Begriff der quidditas entis verschwunden ist. Daraus folgt für den Begriff der realitas, dass er auf die messbaren virtutes eingeschränkt wird". 12

Dieses Resultat kann meines Erachtens weiter bestätigt werden, wenn man berücksichtigt, dass sich in Kants Werken nicht nur zwei Realitätsbegriffe, sondern auch zwei Gradbegriffe gegenüberstehen. Die realitas phaenomenon hat insofern positive Bedeutung, als sie einen „Grad des Einflusses auf den Sinn“ (B208) ausüben kann, wie z. B. wärmere Körper stärkere Empfindungen erzeugen können als lauere. Realität ist daher „das obiect der Empfindung in abstracto“, Negation ist „der Mangel derselben“ (AA 18:56, Refl. 4999; vgl. auch AA 389: 4817). Realitas noumenon ist jedoch die Realität als Objekt des reinen Verstandes. Sie bedeutet etwas positives, insofern sie keinen Widerspruch enthält und das, was sie nicht ist, die Negation, von sich selbst sozusagen fernhält. In dieser Perspektive hat jedes Ding nur etwas Reales und „fehlet doch immer auch etwas, und es ist also kein vollständiges Ding“ (AA 28:1013). Es besitzt also nur einen gewissen Grad an Realität im Vergleich mit dem Wesen, „das alle Realität hat“ (AA 28:1013), dem nichts fehlt und das deswegen vollständig und vollkommen ist.

In beiden Fällen übernimmt Kant Begriffe, die eine lange Tradition hinter sich haben: 1. Eine naturwissenschaftliche Definition von Grad, die sich auf Temperatur, Geschwindigkeit usw. bezieht, die, wie man sagen könnte, als gradus rationis (vgl. AA 28:377, Refl. 5891; AA XXIX, 834), als Grad der Wirksamkeit verstanden werden kann, d.h. vor allem als Fähigkeit, eine stärkere oder schwächere Empfindung hervorzurufen. 2. Eine metaphysische Auffassung vom Gradbegriff als gradus perfectionis: „Die Realität oder die Dingheit ist, daß etwas als ein Ding vollkommen sey“ (AA 28:211). Der realitas phaenomenon wird die erste Form von Grad zugeschrieben; die zweite Form hat nur Bedeutung im Bereich des Intelli-

12 Friedrich Delekat, Immanuel Kant. Historisch-kritische Interpretation der Hauptschriften, 2 ed. (Heidelberg,: Quelle \& Meyer, 1963), 128. Vgl. z.B. diese Reflexion: „Denn alle Dinge ( ${ }^{\mathrm{g}}$ als Erscheinungen) haben eine Größe: extensive und intensive. $\left({ }^{\mathrm{g}}\right.$ Dadurch bekommt mathematik obiective realitaet. Sie geht nicht auf entia rationis). [...] Alle Dinge als gegenstande des reinen Verstandes haben auch eine, nämlich Metaphysische, aber keine transscendentale Größe, weil sie dort als Dinge überhaupt mit allen andern, hier aber als Begrif ( $\left({ }^{g}\right.$ des Dinges) mit dem Wesen des Dinges selbst verglichen werden“ (AA 18:241f., Refl. 5589). 
giblen, d.h. für die realitas noumenon, ein Bereich, der nach Kants Lehre aber der Erkenntnis nicht zugänglich ist. „Die Quantitas phaenomenon zu erkennen und zu bestimmen lehrt die Mathematik, die Qvanta noumena nur die philosophie. Allheit, Uneingeschraenktheit. - Realitaet, Grad etc etc“ (AA 28:679, Refl. 4698).

\subsubsection{Die naturwissenschaftliche Auffassung von Grad}

Wenn man Kants Auffassung von der hier bezeichneten naturwissenschaftlichen Bedeutung des Begriffs von ,intensiver Größe“ oder Grad in Betracht zieht, so scheint es auf den ersten Blick, als ob Kant nichts neues zu behaupten versucht, sondern die klassische Definition der intensiven Größe als quantitas qualitatis einfach wiederholt. ${ }^{13}$ Eine intensive Größe unterscheidet sich zunächst von einer extensiven: ${ }^{14}$

wenn ich einen Keßel und einen Fingerhut voll warmes Waßer nehme, so ist erstes extensiv größer als das letzte, ist aber das Waßer im Keßel nur lau und im Fingerhut siedend so ist dies lezte wieder intensiv größer als das 1ste (AA 28:425).

Diese Unterscheidung kann - modern gesprochen - wie folgt präzisiert werden: Die extensive Größe ist eine addierbare Größe; die intensive eine nicht-addierbare. Wenn man z.B. ein Volumen Wasser bei Zimmertemperatur mit einem ebensolchen Volumen Wasser mischt, dann hat man zwar das doppelte Volumen Wasser, dieses hat aber immer noch die gleiche Temperatur. Das Volumen ist ein Beispiel einer extensiven, die Temperatur einer intensiven Größe.

Eine extensive Größe ist also jene, in der „die Vorstellung der Theile die Vorstellung des Ganzen möglich macht“ (B203). Bei der intensiven Größe kann dagegen „die Vielheit nur durch Annäherung zur Negation = 0“ vorgestellt werden

13 „[Q]uantitas qualitatis est gradus“ (AA 4:309, Anm.). In Baumgartens Metaphysica kann man die gleiche Definition finden: (Baumgarten, Metaphysica, §246). Auch Christian Wolff definiert in seiner Ontologia den Grad als „quantitas qualitatum“ (Wolff, „Philosophia prima sive Ontologia (1730),“ §747). Mehr dazu Antonio Moretto, Dottrina delle grandezze e filosofia trascendentale in Kant (Padua: Il Poligrafo, 1999), $259 \mathrm{ff}$.

14 Vgl. dazu Jonathan Bennett, Kant's Analytic (Cambridge: Cambridge University Press, 1966), 170; Gernot Böhme. „Über Kants Unterscheidung von extensiven und intensiven Größen.“ in Kant Studien 65 (1974), 239-58; Paul Guyer, Kant and the Claims of Knowledge (Cambridge: Cambridge U. Press, 1987), 200; Abhaya C. Nayak und Eric Sotnak, „Kant on the Impossibility of Soft Sciences." in Philosophy and Phenomenological Research 75, Nr. 1 (1995), 133-51. 
(B210). Intensive Größen sind nämlich Niveaugrößen, ${ }^{15}$ die durch das Gleichgewicht verglichen werden können: Die gleiche Temperatur haben jene Körper, deren Temperatur sich nicht ändert, wenn sie sich berühren. Ein Körper ist dagegen wärmer als ein anderer, wenn er die Tendenz hat, an den anderen Wärme abzugeben, bis die Temperaturdifferenz gleich null geworden ist, d. h. bis alles wiederum im Gleichgewicht ist. ${ }^{16}$

Die extensive Größe wird also durch die Menge der Teile, die sie zusammensetzen, gemessen, die intensive erst durch die Wirkungen, die sie hervorruft, etwa die Ausdehnung von Quecksilber. So schreibt Kant etwa: „Die intensive Größe ist die Größe des Grundes, und die extensive Größe ist die Größe des aggregats“ (AA 28:562) ${ }^{17}$.

So kann man z.B. nicht sagen, die Wärme bestehe aus Lauigkeiten, man bestimmt also ihre Größe nicht nach den Theilen, welche sie enthält, sondern nach den Wirkungen, die sie hervorbringt, z. B. daß sie die Körper ausdehnt und man kann ihr daher nicht eine eigentliche Größe beilegen, sondern einen Grad (AA 18:322, Refl. 5663).

Beispielsweise kann man nicht sagen, „daß eine gegebene Geschwindigkeit aus kleinern und eine Schnelligkeit aus Langsamkeiten ebenso bestehe, wie ein Raum aus kleineren“ (AA 4:493; vgl. 14:145). Ein Aggregat vieler langsamer Bewegungen erzeugt keine schnellere Bewegung. Die „Theile der Geschwindigkeit sind nicht außerhalb einander, wie die Theile des Raumes“, „so muß der Begriff ihrer Größe, da sie intensiv ist, auf andere Art construirt werden, als der der extensiven Größe des Raumes“ (AA 4:493). Ein bewegter Körper besitzt nämlich in jedem unteil-

15 „Der Temperaturbegriff ist ein Niveaubegriff wie die Höhe eines Schweren, die Geschwindigkeit eines bewegten Körpers, das Elektrische, das Magnetische Potential, die chemische Differenz" Ernst Mach, Die Principien der Wärmelehre. Historisch-kritisch entwickelt (Leipzig: Barth, 1900, 2. Auflage), 57.

16 „Auf diesen Fuß, wenn das Feuerelement unter den Körpern in einem gewissen Raum im Gleichgewichte ist, so sind sie verhältnißweise gegen einander weder kalt noch warm. Ist dieses Gleichgewicht gehoben, so ist diejenige Materie, in die das Elementarfeuer übergeht, verhältnißweise gegen den Körper, der dadurch desselben beraubt wird, kalt, dieser dagegen heißt, in so fern er in jene Materie diese Wärme überläßt, in Ansehung derselben warm. Der Zustand in dieser Veränderung heißt bei jenem Erwärmung, bei diesem Erkältung, bis alles wiederum im Gleichgewichte ist“ (AA 2:185).

17 „Die Größe eines Grundes heißt Grad“ (AA 17:699, Refl. 4756); „Qvantitaet [...] intensive: der Grad, d.i. die Größe als Grund von viel und großen Folgen“ (16:190, Refl. 1999); „Die Größe eines Gantzen ist extensiv, | Die Größe eines Grundes ist intensiv oder Grad“ (AA 17:536, Refl. 4411); „Die Größe eines Dinges als aggregats ist extensiv, die Größe eines Grundes ist instensiv“ (AA 18:705, Refl. 6399). 
baren Augenblick ein bestimmtes „Moment der Bewegung“, ${ }^{18}$ das keine Bewegung ist, sondern bloß als „das Bestreben, einem Körper eine gewisse Geschwindigkeit mitzutheilen“, das „nicht als extensive, sondern als intensive Größe“ angesehen werden muss (AA 14:496, Refl. 67).

„Grad“ bedeutet also in diesem Zusammenhang etwa „Grad von Wirksamkeit“ ${ }^{19}$ Indem Kant sich ziemlich vage auf die Sprache der Infinitesimalrechnung seiner Zeit bezieht, nennt er ihn auch „Moment“, ${ }^{20}$ z. B. das „Moment der Schwere“ (B210), das „Moment der Erwärmung“ (AA 17:536, Refl. 4411) usw.:

Die Erzeugung der realitaet hat ein Moment ${ }^{21}$ [...] Wie ein verschiedenes Moment verschiedene Grade der Geschwindigkeit erzeugt, so verschiedenheit des Eindruks verschiedenen Grad der Empfindung (AA 18:239, Refl. 5582).

Eine solche „Wirkungsfähigkeit“ kann sich also entweder auf die „Empfindung“ oder auf die ,andere[...] Realität in der Erscheinung“ (B210) auswirken.

Die realitas phaenomenon, sofern ihr ein Grad oder eine intensive Größe zugeschrieben wird, scheint also von Kant als etwas charakterisiert zu sein, das die Fähigkeit besitzt, auf andere Realitäten oder auf unsere Sinnesorgane gewisse Wirkungen auszuüben (ein wärmerer Körper kann eine stärkere Empfindung erzeugen oder ein Metall mehr ausdehnen usw.). In den „Anticipationen der Wahrnehmung“ wird aber der „Grad der Realität als Ursache“(B210) nur beiläufig behandelt, „denn“, so Kant, „mit der Causalität habe ich für jetzt noch nicht zu thun“ (B210). Der Bezug auf Empfindung scheint also Kant die Möglichkeit zu bieten, die Idee einer solchen „Wirkungsfähigkeit“ auszudrücken, bloß als „Grad des Einflusses auf den Sinn“ (B208), ohne das Problem der Verbindung zwischen Ursache und Wirkung in Betracht ziehen zu müssen.

18 „Die Größe der Geschwindigkeit (in einem Augenblick) ist die Größe des moments der Bewegung, wodurch nemlich ein Raum in einer Zeit kan zurückgelegt werden“ (AA 17:536, Refl. 4411).

19 Vgl. dazu Daniel Warren, Reality and Impenetrability in Kant's Philosophy of Nature (New York: Routledge, 2001), $22-30$.

20 „[S]o nennt man den Grad der Realität als Ursache ein Moment, z. B. das Moment der Schwere“ (B210).

21 Vgl. auch diese Stelle: „Grad der realität. Erzeugung einer realitaet aus 0, d.i. moment der Erzeugung“ (AA 17:536, Refl. 4411). 


\subsubsection{Die metaphysische Auffassung von Grad}

Wie eben erwähnt, kann aber auch der realitas noumenon ein Grad zugeschrieben werden, aber bloß im metaphysischen Sinne, im Sinne eines Grades der Vollkommenheit: „Die metaphysische Vollkommenheit besteht also im Grade der Realität“ (AA 28:211). Kant greift in diesem Zusammenhang auf die Auffassung zurück, nach welcher die Vollkommenheit einer Sache durch ihre Nachbarschaft (propinquitas) zu dem vollkommensten Ding definiert wird, welches den höchsten Grad an Vollkommenheit besitzt (gradus summus); die Unvollkommenheit durch den Abstand (distantia) von dem vollkommensten Wesen, ens perfectissimus, dem nulla deest perfectio, vel gradus perfectionis:

Ein jedes Ding ist Realität. Die Dingheit, so zu sagen, beruht blos auf Realität. Die Vollkommenheit eines Dinges überhaupt, ist nichts anders, als wie die Größe der Realität. Schlechthin vollkommen ist aber ohne alle Negation, und das ist die größte Realität. Ein ens omnimode reale ist also, im metaphysischen Verstande, das vollkommenste (AA 28:560).

Nach Kant muss also - metaphysisch betrachtet - ,jedes Ding Realität haben [...]; so werden wir unter allen möglichen Dingen uns entweder ein ens realissimum, oder ein ens partim reale, partim negativum vorstellen können“ (AA 18:1013). So ist „[d]er Begriff eines entis realissimi“ identisch mit dem „Begriff eines entis summi; denn alle andere Dinge außer ihm sind partim realia, partim negativa“ (AA 18:1013).

Im Gegensatz zum Bereich der Erscheinungen in der noumenalen Welt gilt also für Kant die Idee von einem gradus perfectionis, nach welchem die Geschöpfe hierarchisch gemäß ihrem gradus entis angeordnet werden können, wie in einer „great chain of being“, das in ens realissimus, gradum realitatis maximum habens gipfelt:

wie können wir die Größe der Realität in den Dingen beurtheilen, wie den Grad ihrer Vollkommenheit bestimmen? Wenn das die Vernunft will; so muß sie sich [...] irgend ein Maximum der Realität denken, von dem sie ausgehen, und alle andere Dinge darnach abwägen kann (AA 28:1004).

Eine solche Auffassung des Realitäts- und des Gradbegriffes findet aber ihren Platz nur in der Welt der „Dinge an sich“, einer Verstandeswelt, die über alle mögliche Erfahrung hinausgeht. Das Urwesen „als das All (omnitudo) der Realität enthaltend (ens realissimum)“ (AA 20:301), welches in der Transscendentalen Dialektik als „Ideal der reinen Vernunft“ erörtert wird, kann nämlich zwar widerspruchlos gedacht werden, aber es kann ihm keine Wirklichkeit zugeschrieben werden. 


\subsubsection{Komparativ vs. Absolut}

Der Unterschied zwischen den eben vorgestellten Auffassungen vom Gradbegriff tritt deutlich hervor, wenn man darauf achtet, dass in den Erscheinungen kein maximum oder minimum zugelassen werden kann, doch aber in der Verstandeswelt, in der wenigstens das maximum der Realität und Vollkommenheit gedacht werden kann. Wie Kant feststellt, macht es nämlich keinen Sinn, von der größten oder kleinsten Geschwindigkeit, Temperatur oder Dichtigkeit zu reden: „Solche Begriffe nennt Leibnitz conceptus deceptores“ (AA 28:561), täuschende Begriffe (vgl. auch AA 16:821, Refl. 3413; AA 17:398, Refl.4051). Im Gegensatz dazu kann der Begriff vom „höchsten Grad der Vollkommenheit“ widerspruchslos gedacht werden, wenn man alle Schranken der endlichen Geschöpfe beiseitelässt; aber der Begriff eines solchen Urwesens ist eine bloße Idee, von der kein Beispiel in der Erfahrung gefunden werden kann: „Alle Größe ist in den Erscheinungen blos comparativ, in Dingen an sich selbst absolut“ (AA 18:705, Refl. 6398). ${ }^{22}$

In den Naturwissenschaften geht es also immer um relative Graddifferenzen; man könnte die absoluten Werte gleichermaßen abnehmen oder zunehmen lassen und alle physikalischen Phänomene würden unverändert bleiben, wenn nur solche Differenzen erhalten blieben. Das „Sein“ im physikalischen Sinne bedeutet nur eine bestimmte „Größe der Realität $(b-a)$ “ (B253), die „ins Unendliche wachsen oder abnehmen oder durch $a$ - $a$ verschwinden“ kann (AA 22: 533). In den Phänomena geht es demnach immer nur um eine bloße „comparative Realitaet“ (AA 18:364, Refl. 5826), der ein bloßes „comparatives nichts“ (AA 14:122, Refl. 40, S.II) gegenübergestellt werden kann: „Realitas est vel comparativa vel absoluta. Realitas phaenomenon non est absoluta“ (AA 17:447, Refl. 4182).

22 Wie Kant selbst bemerkt, geht eine solche Unterscheidung auf Leibniz zurück: „Mais comme nous pensons souvent à des chimeres impossibles, par exemple au dernier degré de la vistesse“ Gottfried Wilhelm Leibniz, Die philosophischen Schriften von Gottfried Wilhelm Leibniz, hrsg. von Carl Immanuel Gerhardt (Berlin: Weidmann, 1875), 4:449. Nach Leibniz, „les formes ou natures, qui ne sont pas susceptibles du dernier degré, ne sont pas des perfections“, dagegen „la plus grande science et la toute-puissance n'enferment point d'impossibilité. Par consequent la puissance et la science sont des perfections, et en tant qu'elles appartiennent à Dieu, elles n'ont point de bornes“ (a.a.0. 427). Kant selbst hat in seinem vorkritischen Versuch einiger Betrachtungen über den Optimismus (1759) geschrieben: „Es giebt Größen, von denen sich keine denken läßt, daß nicht eine noch größere könnte gedacht werden. Die größte unter allen Zahlen, die geschwindeste unter allen Bewegungen sind von dieser Art. Selbst der göttliche Verstand denkt sie nicht, denn sie sind, wie Leibniz anmerkt, betrügliche Begriffe (notiones deceptrices), von denen es scheint, daß man etwas durch sie denkt, die aber in der That nichts vorstellen“ (AA 2:32). Aber Kant bemerkt: „Es ist gar keine größte Zahl möglich, es ist aber ein größter Grad der Realität möglich, und dieser befindet sich in Gott“" (AA 2:32). 
Metaphysisch betrachtet hat dagegen jedes endliche Ding ein „Sein“, insofern ihm ein gewisser Grad der Realität übrig bleibt, im Vergleich zum Wesen, das alle Realitäten enthält: „Denn alle Dinge enthalten Realität, und zwar einen Grad derselben. Dieser wird immer als nur bedingt möglich angesehen, nämlich so fern ich einen Begriff vom realissimo, wovon jener nur die Einschränkung enthält, voraussetze“ (AA 20:330). Das ens realissimus ist also das absolute Grundmaß, wonach man alle anderen Dinge misst, indem ihnen mehr oder weniger Realität fehlt: „Im Noumeno kan ich mir die quantitaet nur durch das Verhaltnis zum illimitato, d.i. dem was omnitudinem enthält, als dem Maasstabe denken, und zwar durch bloße negationen“ (AA 18:380, Refl. 5905). Ein solches Grundmaß ist aber unserer Erkenntnis nicht zugänglich: „Die Metaphysische vollkommeheit ist Grad der Realität, und den können wir nicht schätzen, weil wir keinen Begriff vom höchsten Grad der Realität haben“(AA 29, 1.2:766f.).

\subsection{Realität und Negation}

Die bisherigen Überlegungen sollten gezeigt haben, dass Kant den von der Schulmetaphysik seiner Zeit übernommenen Realitätsbegriff tiefgreifend geändert hat: „die realitas phaenomenon wurde zum Empfindungsgegebenen, die realitas noumenon blieb das bejahende Prädikat, das den Dingen selbst wahrhaft innewohnt““. ${ }^{23}$ Nur in der Transzendentalen Dialektik werden, im Kapitel über das transzendentale Ideal, die Realitäten im traditionellen Sinne als wahrhafte positive Bestimmung betrachtet, die im ens realissimum zusammenbestehen. In den „Anticipationen der Wahrnehmung“ geht es dagegen bloß um die Realität, die nur in unseren Sinnen liegt, die realitas phaenomenon, die näher bestimmt wird als das, was in der Erscheinung der Empfindung korrespondiert. Entsprechend wird die Auffassung des Gradbegriffes als „Grad der Vollkommenheit“, die sich auf das Modell einer hierarchischen Struktur der Verstandeswelt bezieht, durch den bloßen „Grad der Wirksamkeit“ ersetzt, als „<Ursache der> Erzeugung der Empfindung“ (AA 14:119), der nur in der Sinnenwelt ihren Platz findet.

Um die Tragweite dieser Gegenüberstellung richtig einzuschätzen, muss man beachten, dass damit das Verhältnis zwischen Realität und Negation vollkommen umgedeutet wird. In den Erscheinungen ist Realität bloß eine gewisse Graddifferenz $b-a$, die Negation ist das Verschwinden einer solchen Differenz, bis sie gleich null $=0$ wird, wie wenn die entgegengesetzten Größen $+a$ und $-a$ sich gegenseitig aufheben $(a-a=0)$. In Noumena bezeichnen dagegen die Negatio-

23 Maier, Kants Qualitätskategorien, 39. 
nen das, was eine bestimmte Realität nicht ist, wie der Begriff $a$ von nicht $-a$ unterschieden wird, so dass die Wesen sich allein in Bezug auf die positiven Bestimmungen, die sie besitzen, stufenartig anordnen lassen.

Negationen sind deshalb in den Noumena nur durch den metaphysischen Begriff der Schranke definiert, die Negation bedeutet eine Aufhebung, ein nichtSein. Die Negationen haben deswegen keine selbständige Bedeutung, sie drücken nur den Mangel an positivem Sein aus, im Vergleich zum „Wesen, das allen Mangel ausschließe“ (AA 28:994). Bei den Phänomena dagegen sind Negationen nur durch den mathematischen Begriff von „Grenze“ definiert; die Negation bezeichnet das Verschwinden einer quantitativen Differenz, sie ist „eine realitas evanescens oder verschwindende Realität“ (AA 28:426). „Schranke“ bedeutet daher eine bloße negative Bestimmung, das, was fehlt, und deshalb nicht ist; „Grenze“ ist dagegen eine positive Determination, die bloß als ein „verschwindendes quantum“ (AA 18:361, Refl. 5816) anzusehen ist: „Mit dem Begriffe der Schranken, der ein reiner Verstandesbegriff ist, ist verwandt der Begriff der Grenzen, der ein mathematischer Begriff ist. [...] Der Begriff der Grenze gehört nur zu den phaenomenis, aber der der Schranken zu den noumenis“ (AA 28:570).

Die Auffassung der traditionellen Metaphysik kann durch das Prinzip omnes realitates sunt vere positiva zusammengefasst werden, dessen Korrelat nulla negatio est realitas ist. ${ }^{24}$ Alles was ist, insofern es ist, besitzt Realität (omni enti quaedam insit realitas). ${ }^{25}$ Die Negation dagegen ist Aufhebung (remotio), Mangel an Realität (vgl. AA 20:302), und besitzt daher keine ontologische Konsistenz. Dagegen „muss ein Object etwas positives, und kann viel positives oder Vollkommenheit haben“, während „[d]er Begriff de ente omni modo negativo [...] der Begriff eines non entis“ ist (AA 28:1013), weil er einen Widerspruch enthält. ${ }^{26}$

Kants Philosophie übernimmt die Begriffe Realität und Negation von dieser metaphysischen Tradition, aber sie schreibt ihnen eine neue kritische Bedeutung zu, indem sie die Realitäten als bloße Realitäten in der Erscheinung betrachtet: Der Realität ist nicht allein die Negation entgegengesetzt, sondern noch eine andere Realität, die die Folge der anderen aufhebt, „So ist - $a$ für sich etwas Reales, aber [nur ein Grund] in Verbindung mit $a$ ist $a$ - $a$ eine Negation = 0“ (AA 18:647, Refl. 6324); das negative hat also eine positive ontologische Konsistenz, so wie die Realität. $^{27}$

24 Vgl. Baumgarten, Metaphysica, §807.

25 Vgl. A.a.O., §136.

26 ,[0]mni enti quaedam insit realitas, omne ens est reale“, und deshalb ,ens mere negativum est non ens“ (A.a.O., §135).

27 „Realitas phaenomenon ist, was der Empfindung correspondirt. Voller und leerer Raum.| Wir brauchen nicht immer wahre realitaet, an der gar keine Verneinung (non esse) gedacht wird; 
Die Schulmetaphysik, wie sie sich im Leibniz-Wolff'schen System darstellt, behauptete, „daß Realitäten (als bloße Bejahungen) einander niemals logisch widerstreiten“ (B328); jede Realität als realitas noumenon schließt eine andere aus, wie ein Begriff seinen kontradiktorischen Entgegengesetzten ausschließt. Nach Kant ist es aber ,in dem bloßen Begriffe eines Dinges überhaupt [...] auch wirklich so, aber nicht in den Dingen als Erscheinungen“ (B330). Denn hier findet „der reale Widerstreit [...] allerwärts statt“, wie „alle Hindernisse und Gegenwirkungen in der Natur unaufhörlich vor Augen legen, die [...] realitates phaenomena genannt werden müssen“ (B329). War in der ersten logischen Form des Gegensatzes, in dem Versuch einer gedanklichen Verknüpfung zweier logisch entgegengesetzter $a$ und nicht- $a$ das Ergebnis ein Widersinn (nihil negativum), so ist das Resultat seine kontradiktorisch Entgegensetzung von $+a$ und - $a$ eine feste und völlig eindeutige Größenbestimmung, denn die Größe „Null“ (nihil positivum) ist nicht minder bestimmt als irgendeine andere, die durch eine positive oder negative Zahl bezeichnet wird. ${ }^{28}$

\begin{abstract}
Wenn Realität nur durch den reinen Verstand vorgestellt wird (realitas noumenon), so läßt sich zwischen den Realitäten kein Widerstreit denken, d.i. ein solches Verhältniß, da sie, in einem Subject verbunden, einander ihre Folgen aufheben, und 3-3=0 sei. Dagegen kann das Reale in der Erscheinung (realitas phaenomenon) unter einander allerdings im Widerstreit sein und, vereint in demselben Subject, eines die Folge des andern ganz oder zum Theil vernichten, wie zwei bewegende Kräfte in derselben geraden Linie, sofern sie einen Punkt in entgegengesetzter Richtung entweder ziehen oder drücken, oder auch ein Vergnügen, was dem Schmerze die Wage hält (B321).
\end{abstract}

Die Identifikation von Positivität und Vollkommenheit (perfectio est gradus realitatis positivae) sowie von Sein und Gut (omne ens est perfectum et bonum trascendentaliter), die nicht nur die mittelalterliche Metaphysik, sondern auch das Denken der Neuzeit von Descartes über Spinoza und Leibniz bis zu Baumgarten ${ }^{29}$ geprägt hatte, wird damit ein für allemal zerstört.

sondern anfänglich haben wir es auch mit realitatibus phaenomenis zu thun“ (AA 18:361, Refl. 5814).

28 „Zwischen zwey logice oppositis giebt's kein Drittes (tertium non datur); zwischen zwey realiter oppositis aber giebt es ein Drittes (tertium datur)“ (AA 28:549). Kant greift selbstverständlich auf die Resultate seiner vorkritischen Schrift Versuch den Begriff der negativen Größen in die Weltweisheit einzuführen (1763) (AA 2:167-203) zurück, in dem er zwischen einer logischen und einer realen Entgegensetzung unterscheidet.

29 In den Principia philosophiae Cartesianae heißt es: „per perfectionem intelligo tantum realitatem, sive esse“ (Benedictus de Spinoza, Opera, hrsg. von Carl Immanuel Gerhardt (Heidelberg,: C. Winter, 1925), 1:165), und in der Ethica „per realitatem, et perfectionem idem intelligo“ Spinoza, Opera, 2:85. Vgl. dazu Giuseppina Totaro, „Perfectio e realitas nell'opera di Spinoza,“ in 
Traditionell wurde, wie Kant schreibt, „das Gute [als] Realität, das Böse aber nur [als] Einschränkung der Realität“ (AA 28:346; vgl. auch AA 20:282) betrachtet, d. h. als das Nichtgute und deshalb als Nichtsein. Das bonum metaphysicum wurde immer als eine positive Realität betrachtet, mag diese noch so beschränkt sein, während das malum metaphysicum nur als Mangel, als Unvollkommenheit der Geschöpfe wahrgenommen wurde. Dagegen kann für Kant „das Nichtgute auch das positive Böse heißen“ (AA 6:22-23Anm.), so dass „das positiv Böse“ auch „Realitaet“ ist (AA 28:635):

[Leibniz behauptete], daß alle Dinge, metaphysisch betrachtet, aus Realität und Negation, aus dem Seyn und dem Nichtseyn, [...] zusammengesetzt wären, und der Grund einer Negation kein anderer seyn könne, als daß [...] keine Realität da ist, und so brachte er aus allem sogenannten metaphysischen Bösen, in Vereinigung mit dem Guten dieser Art, eine Welt aus lauter Licht und Schatten hervor, ohne in Betrachtung zu ziehen, [...] daß [...] eine Entgegensetzung des Realen (der bewegenden Kraft), gegen ein andres Reale, nämlich einer bewegenden Kraft in entgegengesetzter Richtung [...] Negation seyn könne (AA 20:282).

Die theoretische Tragweite von Kants Resultat ist kaum zu unterschätzen, wie seine Wirkungsgeschichte zeigt. Es ist kein Zufall, dass der klassische deutsche Idealismus Kants Auffassung der Realität des Negativen übernommen und radikalisiert hat. Man denke etwa an die zentrale Rolle, die das Phänomen der „Polarität“ in Friedrich Wilhelm Joseph Schellings Naturphilosophie (1775-1854) und in der Naturphilosophie im Allgemeinen - spielt, insofern es zeigt, dass das Positive und das Negative als bloß relative Bestimmungen betrachtet werden müssen, so wie in einem Magneten ,jeder Punkt Indifferenzpunkt, Pol und positiver oder negativer Pol [ist], je nachdem er bezogen wird“ “. ${ }^{30}$ Aber vor allem hat Schelling ausdrücklich die Auffassung „des Bösen als positiven Gegensatzes“ vertreten, und die „Reduktion desselben auf das sogenannte malum metaphysicum“ oder auf den „verneinenden Begriff der Unvollkommenheit der Creatur“31 abgelehnt.

Lexicon Philosophicum. Quaderni di terminologia filosofica e storia delle idée, hrsg. von Antonio Lamarra und Lidia Procesi (Rom: Edizioni dell'ateneo, 1988), Bd. 3. „Perfectio, de qua quaeris, est gradus realitatis positivae“ (Leibniz, Die philosophischen Schriften von Gottfried Wilhelm Leibniz, Bd. I, 226). Vgl. dazu Albert Heinekamp, „Zu den Begriffen realitas, perfectio und bonum metaphysicum bei Leibniz,“ in Akten des ersten Internationalen Leibniz-Kongresses: Hannover, 14.19. November 1966 (Wiesbaden: Steiner, 1968). So Baumgarten: „Positis entis realitatibus ponitur eius perfectio, hinc realitates sunt bona“ Baumgarten, Metaphysica, §147.

30 Friedrich Wilhelm Joseph von Schelling, Friedrich Wilhelm Joseph von Schellings sämmtliche Werke, hrsg. von Karl Friedrich August Schelling (Stuttgart, Augsburg: J.G. Cotta, 1856), 7:185. 31 A.a.o., 7:367. 
Ein Realist wie Johann Friedrich Herbart (1776-1841) greift dagegen ausdrücklich auf die vorkritische Metaphysik zurück und behauptet, dass den Negationen kein ontologischer Status zugeschrieben werden darf: „Dies begriff die alte Schule wenigstens insofern, dass sie einsah, ein ganz negatives Ding könne nicht sein“. ${ }^{32}$ Die alte Schule aber, „nachdem schon das ganz negative Ding zurückgewiesen war“ ${ }^{33}$ ist in dieser Auffassung nicht völlig kohärent geblieben. Sie ließ nämlich ,in den Dingen ein malum metaphysicum zu, was aus den ihnen beiwohnenden Negationen bestehen sollte“. ${ }^{34}$ Damit unterstellt sie, dass doch einige Negationen irgendwie noch „sein“ könnten, was nach Herbart widersprüchlich ist. Herbart glaubt trotzdem, in der Auffassung der Schulmetaphysik wenigstens „die Spur eines bessern Geistes zu bemerken, die sich in den paradoxen Sätzen findet: omnes ens est unum, verum, bonum. Darin liegt ein zwar misslungenes, und gar nicht festgehaltenes, aber dennoch beachtenswerthes Bestreben“35, das echte Prinzip zu erfassen, dass „die Qualität des Seienden [...] gänzlich positiv oder affirmativ [ist]; ohne Einmischung von Negationen“. ${ }^{36}$

\section{Realität, Grad und intensive Größe des Neukantianismus}

Bisher habe ich nachzuweisen versucht, dass bei Kant ein im naturwissenschaftlichen Bereich geltender Begriff von Grad und intensiver Größe (als Grad der Wirksamkeit) und ein über alle mögliche Erfahrung hinausgehender metaphysischer Begriff (Grad der Vollkommenheit) gefunden werden können. Mit einer gewissen Vereinfachung kann man behaupten, dass beide Aspekte des Problems in der Zeit des Neukantianismus Anlass für zwei unterschiedliche Interpretationsrichtungen waren. Es ist kein Zufall, dass beide direkt oder indirekt auf die sogenannte „Marburger Schule“ des Neukantianismus ${ }^{37}$ zurückgeführt werden können. Es gibt nämlich keine philosophische Schule, die so starken exegetischen und systematischen Wert auf das Problem des Intensiven bei Kant gelegt hat. Kurz

32 Johann Friedrich Herbart, Sämtliche Werke, hrsg. von Karl Kehrbach und Otto Flügel, Neudruck ed. (Aalen: Scientia Verlag, 1964), 8:82.

33 Ebenda.

34 Ebenda.

35 A.a.O., 8:91.

36 A.a.O., 8:83.

37 Zur Geschichte der Marburger Schule vgl. vor allem: Ulrich Sieg, Aufstieg und Niedergang des Marburger Neukantianismus. Die Geschichte einer philosophischen Schulgemeinschaft (Königshausen und Neumann, 1994). 
gefasst kann man sagen, dass Hermann Cohen (1842-1918), der Begründer der Schule, und seine Nachfolger versuchten, Kants Intensitätsbegriff anhand der Geschichte der Naturwissenschaften, vor allem der „Infinitesimalmethode“ zu interpretieren; später versuchten dagegen Denker wie z. B. Heinz Heimsoeth - der interessanterweise ausgerechnet bei Cohen und Paul Natorp in Marburg promovierte -, Kants Verwendung der Begriffe Realität, Grad usw. als Kapitel der Geschichte der Ontologie zu interpretieren.

\subsection{Intensive Größe und unendlich kleine Größe. Hermann Cohen und die Marburger Schule}

Cohens Interesse am Thema des „Intensiven“ bei Kant geht auf sein umstrittenes ${ }^{38}$ Buch Das Princip der Infinitesimal-Methode und seine Geschichte ${ }^{39}$ von 1883 zurück, dessen Resultate in der 1885 erschienenen zweiten Auflage seines klassischen Kants Theorie der Erfahrung ${ }^{40}$ wieder aufgegriffen wurden. Cohen versuchte nachzuweisen, dass Kants Verbindung zwischen intensiver Größe und Realität das Erbe einer langen Tradition ist, die sich durch die gesamte Geschichte der modernen Naturwissenschaft zieht und die in die Entdeckung des Infinitesimalbegriffs mündete. In der Mechanik versuchte man von Galileis ,gradus velocitatis“ bis hin zu Leibniz' , intensio motus', jene dynamische Wirksamkeit, die die physische von der bloß geometrischen Betrachtung der Bewegung unterscheidet, durch bestimmte Begriffe - bei Galilei momento, bei Leibniz etwa conatus, impetus, nisus usw. -, ,infinitesimal' gedanklich zu fixieren. Demgemäß, schreibt Cohen, „haben Galilei wie Leibniz von dem Unendlichkleinen, als einer intensiven Größe, geredet“. ${ }^{41}$ Cohen konnte also in der von Kant diagnostizierten Verbindung zwischen Realität, Grad und Moment „das mechanische Motiv“ erkennen, das „zur Entde-

38 Vgl. die kritischen Rezensionen: Gottlob Frege, „Rezension von Das Prinzip der InfinitesimalMethode und seine Geschichte,“ in Kleine Schriften, hrsg. von Ignacio Angelelli (Darmstadt: Wissenschaftliche Buchgesellschaft, 1967) und Georg Cantor. Rezension von Hermann Cohen, Das Prinzip der Infinitesimal-Methode und seine Geschichte, Deutsche Literaturzeitung 5 (1884), 266 - 8. Vgl. auch die Bemerkungen von Bertrand Russell, The Principles of Mathematics (Cambridge: Cambridge University Press, 1903), $338 \mathrm{ff..}$

39 Hermann Cohen, „Das Princip der Infinitesimal-Methode und seine Geschichte: ein Kapitel zur Grundlegung der Erkenntnisskritik (1883),“ in Ders., Werke, hrsg. von Peter Schulthess (Hildesheim: Olms, 1987), Bd. V/1.

40 Hermann Cohen, „Kants Theorie der Erfahrung (1885; 1918), “ in Ders., Werke, hrsg. von Geert Edel (Hildesheim: Olms, 1987), Bd. I,1.

41 A.a.O., 545. 
ckung der Infinitesimalrechnung entscheidend mitgewirkt“42 hat. Die „Identität der intensiven und der unendlichkleinen Größe“ war nämlich „zu Kants Zeiten eine allgemeine Annahme“, ${ }^{43}$ „,[d]enn nichts anderes als jene Differentialgrösse bedeutet die Intensive Größe“. ${ }^{44}$ Kant habe damit einfach ein Problem philosophisch ausgedrückt, das eine zentrale Rolle in der Geschichte der mathematischen Naturwissenschaft spielte: „In diesem mechanischen Motiv“, so Cohen, „entspricht das Differential einem reinen Grundbegriffe des reinen Denkens, der Kategorie der Realität““. ${ }^{45}$

Cohen glaubte also nachweisen zu können, dass einerseits die Entdeckung des Differentialbegriffs $d x$ durchgesetzt wurde, um jenes Problem zu lösen, das Kant später in der Kategorie der Realität und in den „Anticipationen der Wahrnehmung“ gestellt hatte; damit glaubte Cohen die „logische Begründung des Differentialbegriffs in einem erkenntnistheoretischen Grundsatze“ gefunden zu haben, „und zwar in dem der Kategorie der Realität entsprechenden, mithin in dem Grundsatz der intensiven Größe oder der Antizipationen“..46 Andererseits war aber Cohen auch überzeugt, dass die eigentliche Bedeutung von Kants Realitätskategorie und der „Anticipationen der Wahrnehmung“ erst dann zu verstehen ist, wenn man seine Voraussetzung in der Geschichte der mathematischen Naturwissenschaft untersucht.

In der Einführung des Infinitesimalbegriffs zum Zwecke der Definierbarkeit der intensiven und physischen Dimension der Bewegung liegen also zugleich der „Sinn der Realität und das Geheimnis des Differentialbegriffes“: ${ }^{47}$

Es ist der Sinn der intensiven Grösse: dass sie den Grund und Ursprung der extensiven darstelle, die letztere aus sich hervorgehen lasse; dergestalt, dass in ihr das Reale in derjenigen wissenschaftlichen Zurüstung der Physik übergeben werden kann, welche die InfinitesimalRechnung vollbringt. In der Erzeugungsgrösse, welche, in dem Unendlichkleinen entspringend, den Bewegungsgesetzen materieller Punkte sich darbietet, haben wir dasjenige Reale

42 A.a.O., 544. Cohen zitiert z. B. diese Reflexion von Kant: „Die Erzeugung der realitaet hat ein Moment, des qvanti extensivi ein ( ${ }^{\mathrm{g}}$ qvasi) element: differentiale“ (AA 14:458, Refl. 5582).

43 Cohen, „Das Princip der Infinitesimal-Methode und seine Geschichte: ein Kapitel zur Grundlegung der Erkenntnisskritik (1883),“ 14.

44 Cohen, „Kants Theorie der Erfahrung (1885; 1918),“ 544.

45 Cohen, „Das Princip der Infinitesimal-Methode und seine Geschichte: ein Kapitel zur Grundlegung der Erkenntnisskritik (1883),“ 23.

46 A.a.O., 14.

47 A.a.O., 22. 
zu erkennen, welches wir wissenschaftlicherweise in der Empfindung ansprechen; oder welches, von der Empfindung verkündet, wissenschaftlicher zugänglich wird. ${ }^{48}$

Damit wird das Physische im Gegensatz zum bloß Geometrischen nicht nur wie bei Kant als das charakterisiert, was einen „Grad des Einflusses auf den Sinn“ ausübt, sondern durch eine rein begriffliche Bestimmung zu erfassen, nämlich den Infinitesimalbegriff. Cohen betrachtet das als einen „Triumph des Denkens“49, das seine „schöpferische Souveränität“ gegen Kants „Vorurteil des Gegebenseins“50 gewährleistet. In diesem Sinne liegt, wie Ernst Cassirer bemerkt, „in der Fortbildung [...], die Cohen Kants Grundsatz von den ,Antizipationen der Wahrnehmung gegeben hat“, schon „der Weg, der in seiner Weiterführung zu seiner eigenen systematischen Gestaltung der ,Logik der reinen Erkenntnis ${ }^{651}$ hingeleitet hat“ .52

Cohens Interpretation, obwohl sie von einigen ${ }^{53}$ seiner Schüler gänzlich abgelehnt wurde, wurde von anderen seiner Schüler übernommen ${ }^{54}$ und weiter entwickelt, wobei sie aber jene von Cohen oft behauptete „Gleichwertigkeit des Differentialen und des Intensiven “55 geschwächt haben. Sie haben Cohens Per-

48 Hermann Cohen, „Kants Theorie der Erfahrung“ (1885; 1918),“ 758.

49 Cohen, „Logik der reinen Erkenntnis,“ 33.

50 Mehr dazu bei Helmut Holzhey, Cohen und Natorp, 2 vols. (Basel: Schwabe, 1986) und Andrea Poma, The Critical Philosophy of Hermann Cohen (Albany, NY: State University of New York Press, 1997).

51 Cohen, „Logik der reinen Erkenntnis,“ 33.

52 Ernst Cassirer. „Hermann Cohen und die Erneuerung der Kantischen Philosophie (1912).“ Kant Studien 17 (1912), 252 -73. Nachdruck in Ernst Cassirer, „Aufsätze und kleine Schriften (1902 -1921), “ in Ders., Gesammelte Werke, hrsg. von Marcel Simon (Hamburg: Meiner, 1999), Bd. 9, 126.

53 Andere Schüler von Cohen folgten ihm dagegen nicht. August Stadler, einer der ersten Anhänger von Cohens Kantinterpretation, der zwar in seinen ersten zwei Kant-Büchern (August Stadler, Die Grundsätze der reinen Erkenntnisstheorie in der Kantischen Philosophie: Kritische Darstellung (Leipzig: Hirzel, 1876)) sogar zu ihrer Weiterentwicklung beigetragen hatte (vgl. dazu Marco Giovanelli, August Stadler interprete di Kant (Napoli: Guida, 2003)), kritisierte ausdrücklich, den Standpunkt der „die intensive Größe als solche dem Differential entsprechen lässt“ (August Stadler, Kants Theorie der Materie (Leipzig: Hirzel, 1883), 40. Sehr kritisch drückte sich auch der bei Cohen promovierte Ferdinand August Müller aus in Ferdinand August Müller, Das Problem der Continuität im Mathematik und Mechanik: historische und systematische Beiträge (Elwert: Marburg, 1886), 96, Anm..

54 Vgl. insbesondere Stadler, Kants Theorie der Materie und Müller, Das Problem der Continuität im Mathematik und Mechanik: historische und systematische Beiträge .

55 Cohen, „Das Princip der Infinitesimal-Methode und seine Geschichte: ein Kapitel zur Grundlegung der Erkenntnisskritik (1883),“ 15. 
spektive vor allem als einen Beitrag zur Geschichtsschreibung der Naturwissenschaften betrachtet, ohne damit den Anspruch zu haben, jene von Cohen angestrebte „erkenntnisskritische[...] Begründung des Differentialbegriffs“"56 $\mathrm{zu}$ leisten

Besonders interessant ist in dieser Hinsicht das Beispiel von Kurd Laßwitz (1848-1910), Autor einer berühmten Geschichte der Atomistik (1890) (aber auch Schöpfer der deutschsprachigen science fiction), ${ }^{57}$ der in enger Verbindung mit Cohen stand, obwohl er kein echtes Mitglied der Marburger Schule war. ${ }^{58}$ Laßwitz hat - und deutlicher noch als Cohen - am Beispiel Galileis gezeigt, dass das Grundproblem der entstehenden modernen Bewegungslehre darin bestand, in jedem unausgedehnten Zeitmoment, in dem keine Ortsveränderung und deswegen Bewegung stattfindet, noch die „dynamische Wirkungsfähigkeit der bewegten Körper“, ${ }^{59}$ das „intensive der Bewegung“, ${ }^{60}$ seine „Realität“61 zu bestimmen. Das war der Anlass für die Entdeckung des Infinitesimalbegriffs, wie er sich zuerst in Galileis „Momentbegriff“ ausdrückte.

Ernst Cassirer (1874-1945) scheint in seinem ersten Buch Leibniz' System in seinen wissenschaftlichen Grundlagen (1902) in Bezug auf Leibniz eine ähnliche Meinung zu vertreten. Dieser habe gezeigt, dass man, um Bewegung nicht nur als phoronomische Ortsveränderung, sondern als physikalisches Objekt zu definieren, etwas braucht , das „zur bloßen Ausdehnung hinzukommt, ja ihr vorangeht“ (praeter extensionem, imo extensione prius), ${ }^{62}$ etwas, das der Bewegung „als intensiver Realität, Tendenz und Streben (conatus, nisus, tendentia)“ zukommt, „so daß sie Wirkung ausübt, sofern sie nicht durch entgegengesetzte Tendenzen kompensiert wird“ “. ${ }^{63}$ Es ist dieses dynamische Prinzip, das ,als eine Ausführung und bestimmtere Gestaltung des Differentialbegriffs“"64 verstanden werden kann.

56 A.a.O., 26.

57 Vgl. vor allem den Roman Kurd Laßwitz, Auf zwei Planeten : Roman in 2 Büchern (Leipzig: Elischer, 1913).

58 Die Diskussion mit Laßwitz ist in Briefen belegt, die in Holzhey, Cohen und Natorp, II, 166 201 veröffentlich sind.

59 Kurd Laßwitz, Geschichte der Atomistik (Hamburg-Leipzig: Voss, 1890), II, 5.

60 A.a.O., II, 6.

61 Ebenda.

62 Gottfried Wilhelm Leibniz, Mathematische Schriften, hrsg. von Carl Immanuel Gerhardt (Hildesheim: Georg Olms, 1971), 6:100.

63 Ernst Cassirer, „Leibniz System in seinen wissenschaftlichen Grundlagen,“ in Ders., Gesammelte Werke, hrsg. von Marcel Simon (Hamburg: Meiner, 1998), Bd. 1, 300.

64 A.a.O., XI. 
Noch etwa zwanzig Jahre später hat Cassirer in seiner Schrift über die Relativitätstheorie ${ }^{65}$ die diesbezügliche Position der Marburger Schule so zusammengefasst:

In dem Maß der Bewegung, das sie entdeckt und begründet, in dem Differentialquotienten des Weges nach der Zeit, glaubt Galileis Physik nun zugleich zum Kern alles physischen Seins vorgedrungen zu sein, glaubt sie die intensive Realität der Bewegung definiert und erfaßt zu haben. Durch diese Realität unterscheidet sich die dynamische von der bloß phoronomischen Betrachtung [...] Auch Leibniz steht in seiner Grundlegung der Dynamik durchaus auf diesem Standpunkt. [...] Die Bewegung als bloße Ortsveränderung in rein phoronomischem Sinne gefaßt - so erklärt er - bliebe selbst etwas rein Relatives; zum Ausdruck einer eigentlichen, einer physischen wie metaphysischen Realität wird sie erst, indem wir ihr ein inneres dynamisches Prinzip [...] hinzufügen. ${ }^{66}$

Diese Rekonstruktion Cassirers, wenn sie den Sinn der Cohen'schen Auffassung deutlicher und präziser ausdrückt, stimmt mit ihr nur scheinbar überein. Schon im ersten Band von Das Erkenntnisproblem in der Philosophie und Wissenschaft der neueren Zeit (1906) vertritt Cassirer in Bezug auf Galilei zwar, wie Cohen, die Meinung, dass mit dem „Begriff des ,Moments“ [...] das erste typische Beispiel des Infinitesimalen entstanden ist“, welches also „seine erste Klärung und Fixierung im Begriff der Geschwindigkeit gefunden“67 hat. Das bedeutet aber für Cassirer, dass „nicht das Differential des Raumes, ja auch nicht das der Zeit von sich allein aus den Weg weisen konnte“, sondern dass „der Begriff des Differentialquotienten [...] den geschichtlichen und logischen Ausgangspunkt“ bildete. ${ }^{68}$ Der Akzent wird also nicht, wie bei Cohen, auf das Differential $d x$ und auf seine vermutete Verbindung mit der intensiven Größe, sondern auf den Differentialquotienten $\frac{d y}{d x}$ gelegt.

Das ist nicht einfach eine notwendige technische Präzisierung, sondern Zeichen einer divergierenden philosophischen Grundeinstellung. Nicht die Erzeugung der extensiven Größe aus der ,intensiven Größe des Differentials“69 ist

65 Vgl. Ernst Cassirer, „Zur Einsteinschen Relativitätstheorie: erkenntnistheoretische Betrachtungen,“ in Ders., Gesammelte Werke, hrsg. von Reinold Schmücker (Hamburg: Meiner, 2001), Bd. 10.

66 A.a.O., 15.

67 Ernst Cassirer, „Das Erkenntnisproblem in der Philosophie und Wissenschaft der neueren Zeit, 1, “ in Ders., Gesammelte Werke, hrsg.von Tobias Berben (Hamburg: Meiner, 1999), Bd. 2, XIII, 562 S., 356.

68 Ebenda.

69 Cohen, „Kants Theorie der Erfahrung (1885; 1918),“ 544. 
nämlich relevant, sondern die funktionelle Abhängigkeit zweier veränderlicher Größen untereinander, die in ihrer Geltung völlig ungeschmälert bleibt, wenn man ihre extensiven Größenwerte jeweils verschwinden lässt. ${ }^{70}$ Der Differentialquotient, wie Cassirer übrigens schon in seinem ersten Leibniz-Buch schreibt, ist damit der deutlichste „mathematische Ausdruck für die Selbständigkeit und Ursprünglichkeit der Relation gegenüber dem Einzelgebilde, das aus ihr gewonnen wird“. ${ }^{71}$ Der Vorrang des Intensiven über das Extensive, der die zentrale Rolle in Cohens Werk spielt, wird damit durch jenen der Relation über die Relationsglieder ersetzt, der zum Grundproblem von Cassirers wissenschaftstheoretischem Hauptwerk von 1910 Funktionsbegriff und Substanzbegriff ${ }^{72}$ wurde.

\subsection{Heinz Heimsoeth und die ontologische Interpretation des Intensitätsbegriffs}

In den 1920er Jahren wird das Bild von „Kant als Erkenntnistheoretiker“, das in der Marburger Schule seine effektivste Systematisierung fand, allmählich ersetzt durch das fast entgegengesetzte Bild von „Kant als Metaphysiker“. ${ }^{73}$ Heinz Heimsoeth (1886-1975) gilt, wenn nicht als Initiator, so doch sicher als einer der

70 Für diese Interpretation vgl. auch Paul Natorp. „Quantität und Qualität in Begriff, Urteil und gegenständlicher Erkenntnis.“ Philosophische Monatshefte 27 (1891), 1-32; 129-60 und Paul Natorp, Die logischen Grundlagen der exakten Wissenschaften (Leipzig; Berlin: Teubner, 1910). 71 Cassirer, „Leibniz System in seinen wissenschaftlichen Grundlagen,“ 161.

72 Vgl. natürlich Ernst Cassirer, „Substanzbegriff und Funktionsbegriff. Untersuchungen über die Grundfragen der Erkenntniskritik,“ in Ders., Gesammelte Werke, hrsg. von Reinold Schmücker (Hamburg: Meiner, 1998), Bd. 6, 161.

73 Das ist der Titel der bekannten Monographie von Max Wundt: Max Wundt, Kant als Metaphysiker. Ein Beitrag zur Geschichte der deutschen Philosophie im 18 Jahrhundert (Stuttgart: Enke, 1924). Im Bezug auf die metaphysischen Kantinterpretationen vgl. Gottfried Martin, „Die deutsche ontologische Kantinterpretation, “ in Gesammelte Abhandlungen (Köln: Kölner Universitätsverlag, 1961), in dem Martin die Interpretationen von Heimsoeth, Hartmann, Heidegger und seine eigene darstellt. Gerhard Funke. „Der Weg zur ontologischen Kantinterpretation.“ in Kant-Studien 62, no. 1 - 4 (1971), 446 - 66 wurde 1971 zum 85. Geburtstag Heinz Heimsoeths publiziert und ist vor allem dessen Werk gewidmet. In Gerhard Funke, „Die Wendung zur Metaphysik im Neukantianismus des 20. Jahrhunderts,“ in Von der Aktualität Kants, hrsg. von Gerhard Funke (Bonn: Bouvier, 1979) wird die Tendenz zur Metaphysik schon im späten Neukantianismus nachgewiesen. Für einen Vergleich mit der Neukantischen Interpretation vgl. auch Helmut Holzhey, „Zu Natorps Kantauffassung, “ in Materialien zur Neukantianismus-Diskussion, hrsg. von Hans Ludwig Ollig (Darmstadt: Wiss. Buchges., 1987). 
Protagonisten der metaphysischen Kantinterpretation, ${ }^{74}$ obwohl er, was in diesen Zusammenhang besonders bedeutsam ist, 1911 bei Hermann Cohen und Paul Natorp in Marburg promoviert hatte. ${ }^{75}$ Die Bedeutung von Heimsoeths Ansatz besteht darin, dass es ihm besser als etwa Max Wundt oder Friederich Paulsen gelungen ist, die vom Marburger Neukantianismus verdrängten ,treibenden metaphysischen Motive“ der kantischen Philosophie hervorzuheben, ohne den „kritische[n] Kant hinter dem metaphysischen [...] verschwinden“ zu lassen. ${ }^{76}$ Das tritt meines Erachtens besonders deutlich zutage, wenn man seine Interpretation der Qualitätskategorien und der intensiven Größe in Betracht zieht.

„Hermann Cohens Interpretationsinteresse“, schreibt Heimsoeth, habe versucht, „Kants [...] Realitätskategorie [...] zu erhellen in Richtung auf der Grundlegung der mathematischen Naturwissenschaften“ ${ }^{* 77}$ Obwohl Heimsoeth die Untersuchung dieses naturwissenschaftlichen Hintergrundes von Kants Intensitätsbegriff durchaus positiv bewertet, bemerkt er gleichzeitig, dass gerade bei den Qualitätskategorien eine solche Auffassung unzureichend erscheint: „Von Anliegen der Mathematischen Naturwissenschaft und Naturphilosophie überhaupt kann man wohl auf die Quantitätsbegriffe oder auf Kausalität und Wechselwirkung, auch auf Möglichkeit und Notwendigkeit, aber kaum auf ,Realität‘ (sofern man darunter wirklich versteht, was mit der Kategorie gemeint ist), auf Negation und Limitation"78 kommen.

Nach Heimsoeth sind nämlich Realität und Negation „fundamentale ontologische Begriffe der Tradition“. ${ }^{79}$ Schon in der Kantforschung des späten 19. Jahrhunderts war aber „die ontologische Tradition, in welcher Kant aufwuchs und immer stand, in solchen Dingen außer Sicht gekommen““ ${ }^{80}$ Erst in den 1930er

74 Vgl. dazu Hinrich Knittermayer. „Zu Heinz Heimsoeths Kantdeutung.“ in Kant Studien 49, no. 2 (1957-8), 293-311.

75 Über Heimsoeths Zeit in Marburg vgl. vor allem den Briefwechsel mit Nicolai Hartmann (Frida Hartmann und Renate Heimsoeth, Hrsg., Nicolai Hartmann und Heinz Heimsoeth im Briefwechsel (Bonn: Bouvier, 1978)). Über den Prozess der Distanzierung vgl. vor allem die Briefe an Hartmann vom 22. Dezember 1911 (79), 10. Januar 1912 (85), 31. März 1912 (101).Vgl. dazu auch Sieg, „Aufstieg und Niedergang des Marburger Neukantianismus. Die Geschichte einer philosophischen Schulgemeinschaft“ $325 \mathrm{ff}$..

76 Gerhard Lehmann. „Kritizismus und kritisches Motiv in der Entwicklung der kantischen Philosophie.“ Kant Studien 68 (1956-7), 29: 29.

77 Heinz Heimsoeth, „Methodenbegriffe der Erfahrungswissenschaften und Gegensätzlichkeiten spekulativer Weltkonzeption (1970),“ in Ders., Studien zur Philosophie Immanuel Kants (Bonn: Bouvier, 1970), Bd. II, 55.

78 Heinz Heimsoeth, „Metaphysische Ursprünge und Ontologische Grundlagen (1956), “ in Ders., Studien zur Philosophie Immanuel Kants (Bonn: Bouvier, 1970), Bd. I, 52.

79 Ebenda.

80 A.a.o., 51. 
Jahren wurden in der „höchst lehrreiche[n] Monographie über ,Kants Qualitätskategorien“ von Anneliese Maier“ „Kants Zusammenhänge oder Auseinandersetzungen mit der Schultradition seiner Zeit“ wieder ans Licht gebracht, ${ }^{81}$ und damit die Einseitigkeit von Cohens rein wissenschaftstheoretischer Lesart aufgezeigt. ${ }^{82}$

Es ist nämlich schwer zu leugnen, dass „,von allen Kategoriengruppen der Kantischen Tafel“ es jene „unter dem Titel der Qualität auftretenden“ sind, „welche am unmittelbarsten auf ihre Herkunft aus der ontologischen Tradition verweisen“. ${ }^{83}$ Heimsoeth ist sich selbstverständlich dessen bewusst, „dass der Grundsatz der Antizipationen der Wahrnehmung faktisch nur von Intensitätsgraden physikalischer realitates handelt"; ${ }^{44}$ diese Restriktion hat aber eine eminent ontologische und metaphysische Bedeutung, die jedoch in enger Verbindung zu den kritischen Motiven kantischen Denkens steht. Die Antizipationen der Wahrnehmung gelten nämlich ,als transzendentaler Qualität-Grundsatz für mögliche Erfahrung (nicht aber über sie hinaus)“. ${ }^{85}$ Die „kritische Wendung“ nämlich scheidet „den eigentlichen Erkenntnisgebrauch derselben im Felde der Erscheinungen, von ihrem Denkbezug auf Intelligibiles ab““ ${ }^{86}$

Für den kritischen Standpunkt kann von intensiven Größen im Sinne von Messbarkeit in einer Kontinuitätsdimension bei den Noumenis nicht mehr die Rede Sein. Der auf den Inbegriff möglicher Erfahrung restringierte Gebrauch der Qualitätskategorien erlaubt auch keine Metaphysik unendlicher Stufe von Geschöpfen bis zum ens illimitatum, wie sie der Dogmatismus vor allem der Leibnizianer behaupten wollte. ${ }^{87}$

Kants Beitrag besteht also nicht mehr - wie Cohen meinte - darin, dass er mit den „Anticipationen der Wahrnehmung“ eines der Grundprobleme der Geschichte der Naturwissenschaften philosophisch formuliert, sondern vielmehr darin, dass im kritischen Denken „[d]as Ineins-Setzen von realitas und perfectio, welches von

81 A.a.O., 52, Anm. 118.

82 Für einen Vergleich zwischen Hermann Cohen und Anneliese Maier siehe Helmut Holzhey, „Das philosophische Realitätsproblem: Zu Kants Unterscheidung von Realität und Wirklichkeit,“ in 200 Jahre Kritik der reinen Vernunft, hrsg. von Joachim Kopper und Wilhelm Marx (Hildesheim, Germany: Olms, 1984).

83 Heimsoeth, „Metaphysische Ursprünge und Ontologischen Grundlagen (1956),“ 51.

84 Heimsoeth, „Methodenbegriffe der Erfahrungswissenschaften und Gegensätzlichkeiten spekulativer Weltkonzeption (1970),“ 128, Anm. 46.

85 A.a.O., 55.

86 Heimsoeth, Studien zur Philosophie Immanuel Kants 1, Metaphysische Ursprünge und ontologische Grundlagen Köln: Kölner Universitätsverlag, 1956), 64.

87 Heimsoeth, „Methodenbegriffe der Erfahrungswissenschaften und Gegensätzlichkeiten spekulativer Weltkonzeption (1970),“ 55. 
Spinoza z. B. ausdrücklich formuliert [...] fragwürdig geworden“ ist. ${ }^{88}$ Während in der Schultradition nur „positive[...] Wesensdeterminationen der Dinge den Charakter der realitates haben“, 89 gehört für Kant dagegen „,[d]as nichts [...] zum Positiven Sachbestand hinzu“, da „die negativen Grössen ihrerseits auch Realitäten sind“.90 „Obgleich Kant keine eigene Ontologie plante“, so ist Heimsoeth dennoch überzeugt, dass ,ihn jedoch eben dieser terminus ontologicus des Nichts nachhaltig beschäftig haben“91 muss.

\section{Konklusion}

In Kants Auffassung der „intensiven“ Größen sind also naturwissenschaftliche und ontologisch-metaphysische Motive enger verbunden als üblicherweise angenommen wird. Wie Gottfried Martin bemerkt hat, „bleibt [es] das geschichtliche Verdienst der Neukantianer, die Bedeutung der Naturwissenschaften, insbesondere der Physik, für Kant aufgezeigt zu haben. Die Kritik der reinen Vernunft ist gewiss eine Wissenschaftstheorie, sie ist gewiss eine Theorie der Erfahrung wie Cohen dies eingehend dargelegt hat".92 Wie aber Martins Arbeit, die übrigens unter dem „Eindruck der Heimsoethschen Arbeiten“ entstand, ${ }^{93}$ versucht hat nachzuweisen, ist die kritische Philosophie „mindestens auch in gleichem Grade eine Ontologie“, ${ }^{94}$ zumindest bezieht sie sich jedenfalls auf ontologische Probleme.

Die Verflechtung dieser beiden Aspekte scheint meines Erachtens in Bezug auf Kants Qualitätskategorien besonders deutlich zutage zu treten. Kants Problemstellung zeigt nämlich, dass das ontologische Modell der traditionellen Metaphysik mit jenem der modernen Naturwissenschaft nicht kompatibel ist. Die traditionelle Metaphysik vertritt, wie eben erläutert wurde, die Idee einer Abstufung der Realität nach verschiedenen Graden von Positivität und Perfektion bis hin zum ens realissimum. In der Welt der Erscheinungen, wie sie die Naturwissenschaften zu erfassen versuchen, ist dagegen alles sozusagen gleichberechtig und befindet sich auf demselben ontologischen Niveau, weil das physische Selbst auf

88 Heimsoeth, Studien zur Philosophie Kants 1, 62.

89 A.a.O., 52.

90 A.a.O., 58.

91 A.a.O., 10.

92 Gottfried Martin, Immanuel Kant: Ontologie und Wissenschaftstheorie (Köln: Kölner Universitätsverlag, 1951), 1951.

93 Knittermayer. „Zu Heinz Heimsoeths Kantdeutung“, 193, Anm. 2.

94 Martin, Immanuel Kant: Ontologie und Wissenschaftstheorie, 7. 
bloße relative Graddifferenzen zurückgeführt werden kann. Ein negatives Ding war in der ersten Auffassung nichts anderes als eine contradictio in adjecto, der sich widersprechende Begriff eines Seienden, das nicht ist; eine negative Wertdifferenz ist dagegen eine völlig eindeutige Größenbestimmung wie eine positive.

\section{Literatur}

Baumgarten, Alexander Gottlieb. Metaphysica. 2. Auflage. Halle: Hemmerde, 1743.

Bennett, Jonathan. Kant's Analytic. Cambridge: Cambridge University Press, 1966.

Böhme, Gernot. „Über Kants Unterscheidung von extensiven und intensiven Größen.“ Kant Studien 65 (1974): 239-58.

Cantor, Georg. Rezension von Hermann Cohen, Das Prinzip der Infinitesimal-Methode und seine Geschichte, Deutsche Literaturzeitung 5 (1884): 266-8.

Cassirer, Ernst. „Aufsätze und kleine Schriften (1902-1921).“ In Ders., Gesammelte Werke, hrsg. von Marcel Simon Hamburg: Meiner, 1999, Bd. 9

Cassirer, Ernst. „Das Erkenntnisproblem in der Philosophie und Wissenschaft der neueren Zeit, 1.“ In Ders., Gesammelte Werke, hrsg. von Tobias Berben Hamburg: Meiner, 1999, Bd. 2, XIII, $562 \mathrm{~S}$.

Cassirer, Ernst. „Hermann Cohen und die Erneuerung der Kantischen Philosophie (1912).“ Kant Studien 17 (1912): 252-73.

Cassirer, Ernst. „Leibniz System in seinen wissenschaftlichen Grundlagen.“ In Ders., Gesammelte Werke, hrsg. von Marcel Simon Hamburg: Meiner, 1998, Bd. 1

Cassirer, Ernst. „Substanzbegriff und Funktionsbegriff. Untersuchungen über die Grundfragen der Erkenntniskritik.“ In Ders., Gesammelte Werke, hrsg. von Reinold Schmücker Hamburg: Meiner, 1998, Bd. 6

Cassirer, Ernst. „Zur Einsteinschen Relativitätstheorie: erkenntnistheoretische Betrachtungen.“ In Ders., Gesammelte Werke, hrsg. von Reinold Schmücker Hamburg: Meiner, 2001, Bd. 10

Cohen, Hermann. „Das Princip der Infinitesimal-Methode und seine Geschichte: ein Kapitel zur Grundlegung der Erkenntnisskritik (1883)." In Ders., Werke, hrsg. von Peter Schulthess Hildesheim: Olms, 1987, Bd. V/1

Cohen, Hermann. „Kants Theorie der Erfahrung $(1885 ;$ 1918).“ In Ders., Werke, hrsg. von Geert Edel Hildesheim: Olms, 1987, Bd. I,1

Cohen, Hermann. „Logik der reinen Erkenntnis.“ In Ders., Werke, hrsg. von Helmhut Holzhey Hildesheim: Olms, 1977, Bd. 6

Delekat, Friedrich. Immanuel Kant. Historisch-kritische Interpretation der Hauptschriften. 2 Auflage. Heidelberg,: Quelle \& Meyer, 1963.

Frege, Gottlob. „Rezension von Das Prinzip der Infinitesimal-Methode und seine Geschichte.“ In Kleine Schriften, hrsg. von Ignacio Angelelli, Darmstadt: Wissenschaftliche Buchgesellschaft, 1967, 99-102.

Funke, Gerhard. „Der Weg zur ontologischen Kantinterpretation.“ Kant-Studien 62, no. 1-4 (1971): 446-66.

Funke, Gerhard. „Die Wendung zur Metaphysik im Neukantianismus des 20. Jahrhunderts.“ In Von der Aktualität Kants, hrsg. von Gerhard Funke, Bonn: Bouvier, 1979, 181-216. 
Giovanelli, Marco. August Stadler interprete di Kant. Neapel: Guida, 2003.

Guyer, Paul. Kant and the Claims of Knowledge. Cambridge: Cambridge U. Press, 1987.

Haas, Bruno. „Kants Qualitätsschematismus.“ In Analysen - Probleme - Kritik 1, hrsg. von Hariolf Oberer, Würzburg: Königshausen und Neumann, 1988.

Hartmann, Frida und Renate Heimsoeth, Hrsg. Nicolai Hartmann und Heinz Heimsoeth im Briefwechsel Bonn: Bouvier, 1978.

Heidegger, Martin. „Die Frage nach dem Ding. Zu Kants Lehre von den transzendentalen Grundsätzen.“ In Gesamtausgabe, Bd. 41, hrsg. von Friedrich-Wilhelm von Herrmann, Frankfurt/Main: Klostermann, 1984.

Heimsoeth, Heinz. Studien zur Philosophie Immanuel Kants. Bd. 1. Metaphysische Ursprünge und Ontologischen Grundlagen., Bonn: Bouvier, 1956.

Heimsoeth, Heinz. Studien zur Philosophie Immanuel Kants. Bd. II. Methodenbegriffe der Erfahrungswissenschaften und Gegensätzlichkeiten spekulativer Weltkonzeption. Bonn: Bouvier, 1970.

Heinekamp, Albert. „Zu den Begriffen realitas, perfectio und bonum metaphysicum bei Leibniz.“ In Akten des ersten Internationalen Leibniz-Kongresses: Hannover, 14.19. November 1966, Wiesbaden: Steiner, 1968.

Herbart, Johann Friedrich. Sämtliche Werke. Hrsg. von Karl Kehrbach und Otto Flügel. Neudruck Auflage. Aalen: Scientia Verlag, 1964.

Holzhey, Helmut. Cohen und Natorp. Basel: Schwabe, 1986.

Holzhey, Helmut. „Das philosophische Realitätsproblem: Zu Kants Unterscheidung von Realität und Wirklichkeit.“ In 200 Jahre Kritik der reinen Vernunft, hrsg. von Joachim Kopper und Wilhelm Marx, Hildesheim: Olms, 1984.

Holzhey, Helmut. „Zu Natorps Kantauffassung.“ In Materialien zur Neukantianismus-Diskussion, hrsg. von Hans Ludwig Ollig, Darmstadt: Wiss. Buchges., 1987, $134-49$.

Knittermayer, Hinrich. „Zu Heinz Heimsoeths Kantdeutung.“ Kant Studien 49, no. 2 (1957-8): $293-311$.

Laßwitz, Kurd. Auf zwei Planeten : Roman in 2 Büchern. Leipzig: Elischer, 1913.

Laßwitz, Kurd. Geschichte der Atomistik. Hamburg-Leipzig: Voss, 1890.

Lehmann, Gerhard. „Kritizismus und kritisches Motiv in der Entwicklung der kantischen Philosophie." Kant Studien 68 (1956-7): 29.

Leibniz, Gottfried Wilhelm. Die philosophischen Schriften von Gottfried Wilhelm Leibniz. Hrsg. von Carl Immanuel Gerhardt. Berlin: Weidmann, 1875.

Leibniz, Gottfried Wilhelm. Mathematische Schriften. Hrsg. von Carl Immanuel Gerhardt. Hildesheim: Georg Olms, 1971.

Mach, Ernst. Die Principien der Wärmelehrehistorisch-kritisch entwickelt. Leipzig: Barth, 1900.

Maier, Anneliese. Kants Qualitätskategorien, Kantstudien. Ergänzungshefte. Berlin: Metzner, 1930.

Martin, Gottfried. „Die deutsche ontologische Kantinterpretation.“ In Gesammelte Abhandlungen, Köln: Kölner Universitätsverlag, 1961.

Martin, Gottfried. Immanuel Kant: Ontologie und Wissenschaftstheorie. Colonel: Kölner Universitätsverlag, 1951.

Moretto, Antonio. Dottrina delle grandezze e filosofia trascendentale in Kant. Padua: Il Poligrafo, 1999. 
Müller, Ferdinand August. Das Problem der Continuität im Mathematik und Mechanik: historische und systematische Beiträge. Marburg, Elwert, 1886.

Natorp, Paul. Die logischen Grundlagen der exakten Wissenschaften. Leipzig, Berlin: Teubner, 1910.

Natorp, Paul. „Quantität und Qualität in Begriff, Urteil und gegenständlicher Erkenntnis.“ Philosophische Monatshefte 27 (1891): 1-32; 129-60.

Nayak, Abhaya C. und Eric Sotnak. „Kant on the Impossibility of Soft Sciences.“ Philosophy and Phenomenological Research 75, Nr. 1 (1995): 133-51.

Poma, Andrea. The Critical Philosophy of Hermann Cohen. Albany, NY: State University of New York Press, 1997.

Russell, Bertrand. The Principles of Mathematics. Cambridge: Cambridge University Press, 1903.

Scaravelli, Luigi. Saggio sulla categoria della realtà. Florenz: Le Monnier, 1947.

Schelling, Friedrich Wilhelm Joseph von. Friedrich Wilhelm Joseph von Schellings sämmtliche Werke. Hrsg. von Karl Friedrich August Schelling. Stuttgart, Augsburg: J.G. Cotta, 1856.

Sieg, Ulrich. „Aufstieg und Niedergang des Marburger Neukantianismus. Die Geschichte einer philosophischen Schulgemeinschaft." Königshausen und Neumann, 1994.

Spinoza, Benedictus de. Opera. Hrsg. von Carl Immanuel Gerhardt. Heidelberg,: C. Winter, 1925.

Stadler, August. Die Grundsätze der reinen Erkenntnisstheorie in der Kantischen Philosophie: Kritische Darstellung. Leipzig: Hirzel, 1876.

Stadler, August. Kants Theorie der Materie. Leipzig: Hirzel, 1883.

Totaro, Giuseppina. „Perfectio e realitas nell'opera di Spinoza.“ In Lexicon Philosophicum. Quaderni di terminologia filosofica e storia delle idée, hrsg. von Antonio Lamarra und Lidia Procesi, Rom: Edizioni dell'ateneo, 1988, Bd. 3, 71-113.

Uehling, Theodore E. „The Forgotten Principle: Kant's Anticipations of Perception.“ In Akten des 5. Internationalen Kant-Kongresses Mainz. April 4-8, 1981, hrsg. von Gerhard Funke, Bonn: Bouvier, 1981.

Warren, Daniel. Reality and Impenetrability in Kant's Philosophy of Nature. New York: Routledge, 2001.

Wolff, Christian. „Philosophia prima sive Ontologia (1730).“ In Ders., Gesammelte Werke, hrsg. von Jean Ecole Hildesheim: Olms, 1962, Bd. II.3

Wundt, Max. Kant als Metaphysiker. Ein Beitrag zur Geschichte der deutschen Philosophie im 18 Jahrhundert: Stuttgart: Enke, 1924. 
